\title{
REVIEW \\ Anticonvulsant medication use for the management of pain following spinal cord injury: systematic review and effectiveness analysis
}

\author{
S Guy ${ }^{1}, S$ Mehta ${ }^{1}$, L Leff ${ }^{2}, \mathrm{R}$ Teasell ${ }^{1,3}$ and E Loh ${ }^{1,3}$
}

Study design: Systematic review and effectiveness analysis.

Objectives: Assess the effectiveness of anticonvulsants for the management of post spinal cord injury (SCI) neuropathic pain.

Setting: Studies from multiple countries were included.

Methods: CINAHL, Cochrane, EMBASE and MEDLINE were searched up to April 2013. Quality assessment was conducted using the Jadad and the Downs and Black tools. Effect sizes and odds ratios were calculated for primary and secondary outcome in the included studies.

Results: Gabapentinoids, valproate, lamotrigine, levetiracetam and carbamazepine were examined in the 13 included studies, ten of which are randomized controlled trials. Large effect size (0.873-3.362) for improvement of pain relief was found in 4 of the 6 studies examining the effectiveness of gabapentin. Pregabalin was shown to have a moderate to large effect (0.695-3.805) on improving neuropathic pain post $\mathrm{SCl}$ in 3 studies. Valproate and levetiracetam were not effective in improving neuropathic pain post $\mathrm{SCl}$, while lamotrigine was effective in reducing neuropathic pain amongst persons with incomplete lesions and carbamazepine was found effective for relief of moderate to intense pain.

Conclusion: Gabapentin and pregabalin are the two anticonvulsants which have been shown to have some benefit in reducing neuropathic pain.

Spinal Cord (2014) 52, 89-96; doi:10.1038/sc.2013.146; published online 3 December 2013

Keywords: anticonvulsant; spinal cord injuries; neuropathic pain; systematic review

\section{INTRODUCTION}

Chronic pain following spinal cord injury (SCI) is common, debilitating and difficult to treat. The estimated number of people with pain following SCI ranges as high as $63-91 \% .{ }^{1}$ Those with pain are significantly impaired by it and they report a significant impact on health perception, function and quality of life. Spinal cord injured patients affected by pain indicate that pain interferes with their daily activities more severely than the injury itself, ${ }^{2}$ and they have a more negative perception of their health than those without pain. ${ }^{3}$

Despite the prevalence of pain after SCI and its impact on function and quality of life, patients with SCI indicate that significant pain reduction and relief is difficult to achieve., ${ }^{2,4,5}$ In a survey of 117 people with traumatic SCI, Jensen et al. ${ }^{4}$ report that first-line treatments (e.g., gabapentin) for chronic pain in SCI are associated with minimal pain relief. Similarly, in a postal survey of 575 persons with SCI, Heutink et al. ${ }^{5}$ document that high levels of pain persist despite treatment.

Pain following SCI can be neuropathic or nociceptive in origin, and neuropathic pain that occurs below the level of injury is particularly difficult to manage. ${ }^{4}$ Anticonvulsants are commonly used for management of neuropathic pain in SCI. ${ }^{4,5}$ Gabapentin and pregabalin, both anticonvulsants, are considered first-line treatment under French guidelines ${ }^{6}$ and a proposed algorithm for management of pain after SCI. ${ }^{7}$ Lamotrigine and other anticonvulsants are considered second/third line in the management of at or below level neuropathic pain after spinal cord injury. ${ }^{6,7}$

Anticonvulsants have pharmacologic effects on various putative pathophysiologic mechanisms underlying the development of neuropathic pain. ${ }^{8,9}$ Mechanisms of action include sodium and calcium channel modulation, GABA inhibition and suppression of neuronal hyperexcitability. ${ }^{9}$ For example, gabapentin inhibits presynaptic calcium channel activation at primary nociceptive afferents in the dorsal horn necessary for central sensitization. ${ }^{8}$ Gabapentin also has a partial effect at the sodium channel; ${ }^{9}$ sodium channel blockade eliminates ectopic impulses from damaged afferent nociceptors that could lead to the development of neuropathic pain. ${ }^{8}$

Although a part of the Canadian Pain Society fourth line recommendations, general neuropathic pain guideline recommendations, previous reviews have noted a lack of evidence for the use of anticonvulsants in the management of below and at-level neuropathic pain after SCI. ${ }^{6,7}$ Evidence for the use of gabapentin and pregabalin is limited and based on small studies. ${ }^{10,11}$

Given the limited evidence on anticonvulsant use for neuropathic pain after $\mathrm{SCI}^{6,7,10}$ and patient surveys that document a lack of

\footnotetext{
${ }^{1}$ Aging, Rehabilitation and Geriatric Care, Lawson Health Research Institute, London, ON, Canada; 2Parkwood Staff Library, St. Joseph's Healthcare, London, ON, Canada and ${ }^{3}$ Department of Physical Medicine and Rehabilitation, University of Western Ontario, London, ON, Canada

Correspondence: Dr E Loh, Physical Medicine and Rehabilitation, Parkwood Hospital, 801 Commissioner's Rd E, London, ON, Canada N6C 5J1.

E-mail: Eldon.Loh@sjhc.london.on.ca
}

Received 19 August 2013; revised 21 October 2013; accepted 23 October 2013; published online 3 December 2013 
perceived effectiveness of these medications, ${ }^{4}$ the purpose of this study was to perform a systematic review and if possible a metaanalysis of the effectiveness of anticonvulsants for the management of post-SCI neuropathic pain. Clarity regarding the effectiveness and potential risks of anticonvulsants for neuropathic pain after SCI is particularly important as neuropathic pain has a profound effect on the well-being of many individuals with SCI.

\section{MATERIALS AND METHODS}

\section{Literature search strategy}

A systematic review of relevant literature was conducted using multiple electronic databases, including CINAHL (1991-22 April 2013), Cochrane Database of Systematic Reviews (2005-March 2013), EMBASE (1947-22 April 2013) and MEDLINE (1946-22 April 2013). Restrictions included: English Language Only, 'Human' and 'NOT Animal'. In addition, the grey literature was searched through clinicaltrials.gov. In order to adequately represent the concepts of anticonvulsants, pain and spinal cord injury, a combination of MeSH, EMTREE and CINAHL Subject Headings and keywords were used. The electronic search strategy is available as Supplementary Information. The references of identified articles were searched for records which may not have been captured through database searches. A decision was made not to hand search journals as the most relevant journals were indexed in the bibliographic databases used. Thus hand searching would have repeated the work already conducted. Conference proceedings were retrieved within EMBASE.

\section{Inclusion/exclusion criteria}

Published studies were selected for analysis if the following criteria were met: (1) examined the role of anticonvulsants; (2) focused on neuropathic pain; (3) contained persons with SCI; (4) described an intervention; (5) participants were 18 years or older; (6) was written in English language; (7) designed as randomized controlled trials (RCT) or observational design; and (8) measured pain as an outcome. Studies were excluded if: (1) it was a review; or (2) there were less than 3 participants with SCI.

\section{Selection of relevant studies}

Following removal of duplicates, two authors (SM and SG) independently reviewed the titles and abstracts retrieved from the electronic search. Where conflict over an inclusion decision arose, a third reviewer (EL) resolved it. Full papers were obtained for records deemed eligible. Figure 1 represents the retrieval and selection of studies.

\section{Study appraisal and data synthesis}

Extraction of data was undertaken by two independent reviewers (SM and SG). A data extraction form was created based on previous systematic reviews of pharmacological treatment of chronic pain in general populations containing information on: sample characteristics, study identification, intervention, drug used, statistical analysis, outcome measures and results. A quality assessment was conducted for all RCTs by two independent reviewers using the Jadad quality assessment measure. ${ }^{12}$ This tool, which has been validated in a population with pain, consists of three items with a five point quality scale; higher scores represent a higher methodological quality. ${ }^{12}$ The Downs \& Black ${ }^{13}$ scale was used by two independent reviewers to assess quality of nonRCTs. This tool contains 27 items yielding a maximum score of 44 . Higher scores reflect a greater level of methodological quality.

\section{Data analysis}

Effect sizes for each intervention were provided where possible. The effect size was calculated as a standardized mean difference for each study by subtracting of the control group from the treatment group and dividing by the pooled standard deviation, representing size and direction of the treatment in each study. Data was analyzed using Comprehensive MetaAnalysis Software, V2. As outcomes were heterogeneous, we could not conduct a pooled effects analysis.
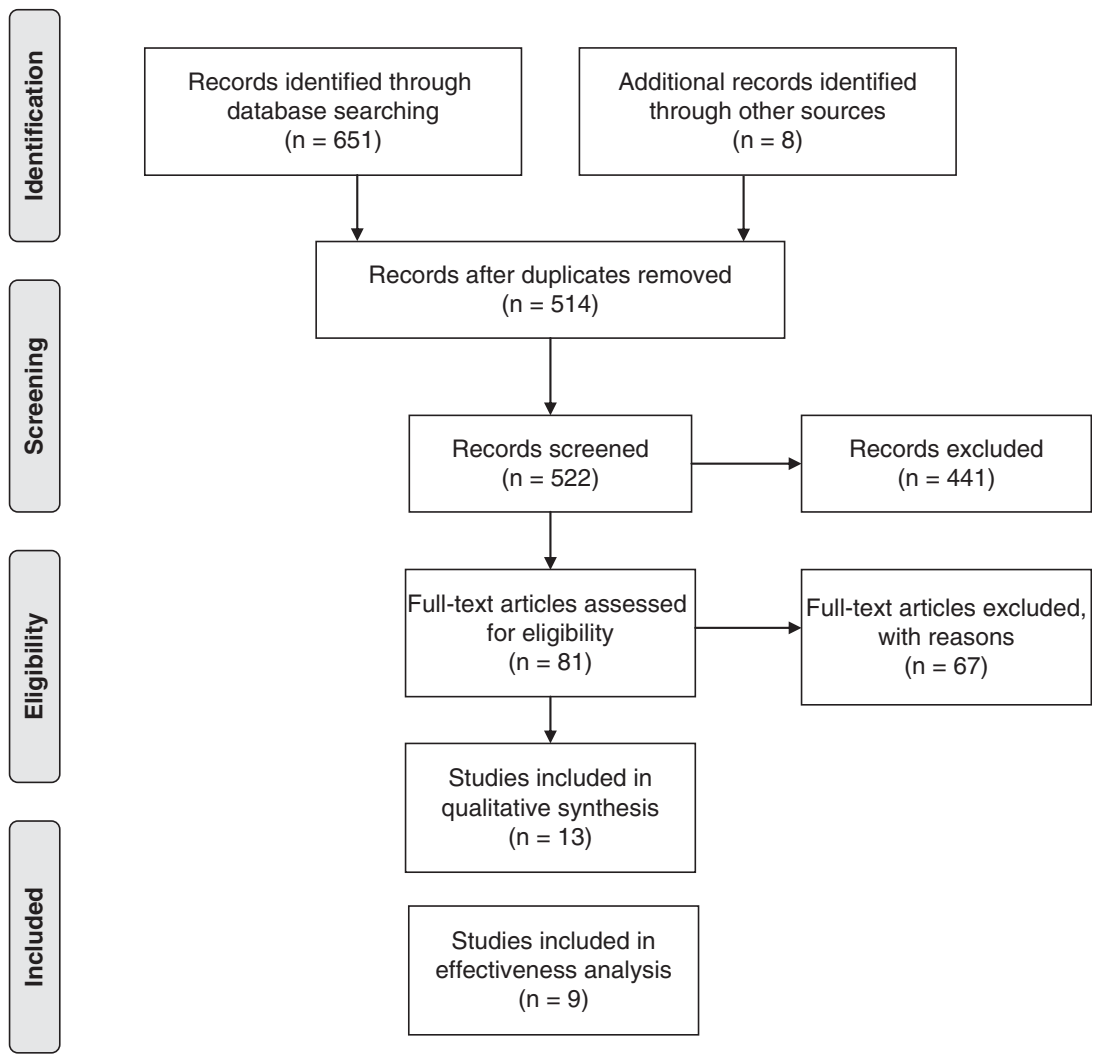

Figure 1 Retrieval and selection of records for inclusion. 
Table 1 Anticonvulsants for spinal cord injury pain studies included in the systematic review

\begin{tabular}{llll}
\hline Study identification & Population (N;M:F) & Drug & Results \\
(author, country) & Type of pain & Intervention &
\end{tabular}

Study design

Outcome measure

Quality assessment

\begin{tabular}{ll}
\hline Ahn et al. & $31 / 31$ SCl \\
Korea & $19: 12$ \\
Pre-post & Neuropathic
\end{tabular}

$D \& B=27 / 44$

\section{Cardenas et al. ${ }^{15}$}

Chile, China,

Columbia, Czech

Republic, Hong Kong

India, Japan,

Philippines, Russian

Federation, USA

RCT

Jadad $=5 / 5$

Drewes et al. ${ }^{16}$

Denmark

Double-blind,

crossover RCT

Jadad $=5 / 5$

Finnerup et al. ${ }^{17}$

Denmark

RCT

Jadad $=5 / 5$

\section{Finnerup et al. ${ }^{18}$}

Denmark

Double-blind,

crossover RCT

Jadad: $5 / 5$

Levendoglu et al. ${ }^{19}$

2004

Turkey

Double-blind,

crossover RCT

Jadad: $3 / 5$

\author{
Putzke et al. ${ }^{26}$ \\ USA \\ Longitudinal \\ observational design \\ D\&B: $24 / 44$ \\ Rintala et al. ${ }^{20}$ \\ USA \\ Double-blind, triple \\ crossover RCT \\ Jadad: $5 / 5$
}

219/219 SCI

176:43

Below level

neuropathic

20/20 SC

15:5

Severe, chronic

central

$30 / 30 \mathrm{SCl}$

18:4

Neuropathic

$36 / 36$ SC

29:7

At and below leve

neuropathic

20/20 SCI

13:7

Neuropathic

\section{$21 / 21 \mathrm{SCl}$ \\ 16:5}

Neuropathic

$38 / 38 \mathrm{SCl}$

$32: 2$

Chronic neuropathic
Gabapentin

$300 \mathrm{mg}$ administered to 2 groups ( $<6 \mathrm{mo}$

pain vs $>6$ mo pain). Dose increased over

18 days to $1500 \mathrm{mg}$, followed by a 5-week maintenance period. If pain score did not decrease, dose increase of 2400-3600 $\mathrm{mg} / \mathrm{day}$.

Pregabalin

Treatment: $150 \mathrm{mg}$ per day pregabalin for 7 days, increased to $300 \mathrm{mg}$ per day on day 8 , $450 \mathrm{mg}$ per day on day 15 and 600mg per day on day 22. 12-week maintenance period followed. Patients tapered off 1 week.

Control: 150-600 mg per day matching

placebo per day for 17 wks.

Duration adjusted average change in pain

(DAAC); change in mean pain score; patient global impression of change score; change in mean pain-related sleep interference score.

Valproate

3-week treatment period started with $600 \mathrm{mg}$

b.i.d. of valproate of placebo followed by

2 -week washout period. New 3-week treat-

ment period followed that.

MPQ (Danish)

Lamotrigine

Treatment: 9-week treatment period starting

with 25 or $100 \mathrm{mg}$ and to $400 \mathrm{mg}$ per day

followed by 2 -week washout period. Drug

doses were decreased if the patient could not

tolerate the $400 \mathrm{mg}$ per day doses. Doses

were not allowed to drop below $200 \mathrm{mg}$ per

day.

Control: Identical placebo.

$M P Q$

Levetiracetam

Two 5-week treatment groups receiving either

levetiracetam or placebo tablets. After a

1 -week washout period, individuals were

crossed over to the 2 nd group. Patients

received $500 \mathrm{mg} \times 2$ for the first week to

$1000 \mathrm{mg} \times 2$ for the second week,

$1500 \mathrm{mg} \times 2$ for the $3 \mathrm{rd}-5$ th week.

NRS

Gabapentin

4-week titration period (gabapentin or placebo) followed by a 4-week stable dosing period, 2-week washout period and then cross over to the alternative treatment plan for 4 wks titration and 4 wks stable dose. VAS, NPS

Gabapentin

Structured interview by telephone or sched uled clinic visit at 6 and 36 mo after trial. NRS

Gabapentin, amitriptyline, diphenhydramine. 6 groups with differing order of drug treatment.

Each drug was administered for 9 wks with 1 washout week before and after each drug treatment, for a total of 31 wks.

The maximum doses were $50 \mathrm{mg}, 3 \times$ per day for amitriptyline; $1200 \mathrm{mg}, 3 \times$ per day for gabapentin; $25 \mathrm{mg}, 3 \times$ per day diphenhydramine (control)

VAS; NRS; CESD-SF
Both groups had lower mean scores for pain and sleep interference score $(P<0.05)$.

Mean pain score decreased more for group 1 than group $2(P<0.05)$.

Mean pain score decreased more for group 1 during wks $2-8$ than for group $2(P<0.05)$.

150-600 mg per day is effective in reducing DAAC in pain over a 16-week period.

DAAC least squares mean for placebo was -1.07

$(0.15)$, for pregabalin $-1.66(0.16)$, with a difference of $-0.59(0.20) \mathrm{Cl}(-0.98$ to -0.21$), P=0.003$.

Pain change from baseline least squares mean for placebo was $-1.22(0.19)$, treatment $-1.92(0.2)$, difference $-0.70(0.25) \mathrm{Cl}(-1.20$ to -0.20$)$, $P=0.0007$

No significant differences between treatment and control.

Trend towards improvement in treatment group.

No significant effect on pain intensity for the total sample $(P=0.11)$

Lamotrigine reduced pain for incomplete lesion group $(P=0.002)$ when compared to the placebo.

No significant improvement in median pain intensity compared to placebo treatment $(P=0.46)$.

No difference in pain relief between the patients treated with drug alone and those with concomitant pain medication.

Pain intensity decreased in both groups $(P<0.000)$ at 4th and 8th week.

Intensity of pain decreased significantly for gabapentin groups $(P<0.001)$.

Intensity of pain differed between the 2 groups at all time periods $(P<0.001)$.

VAS scores indicated was significant pain relief which began at week 2 and continued until week $6(P<0.05)$ VAS scores indicate that pain relief between the 2 groups at the end of the stable dosing periods was $60.7+12.7$ (experimental group) and $10.3+2.8 \%$ (placebo group), $P<0.000$.

14 patients reported favourable response to use of gabapentin - 2 or greater point reduction on pain scale. At second follow-up, 10 of 11 patients continued to report gabapentin was an effect analgesic.

At week 8, amitriptyline was more effective than diphenhydramine $(P=0.35)$ in patients with high baseline CESD-SF scores.

Amitriptyline was not significantly more effective than gabapentin $(P=0.61)$.

Gabapentin no more effective than diphenhydramine $(P=0.97)$. 
Table 1 (Continued)

\begin{tabular}{llll}
\hline Study identification & Population (N;M:F) & Drug & Results \\
(author, country) & Type of pain & Intervention & \\
Study design & & Outcome measure &
\end{tabular}

Quality assessment

Salinas et al 21

Salinas et

Double-blind,

group RCT

Jadad: $5 / 5$

Siddall et al. 22

Australia

Double-blind, parallel-

group RCT.

Jadad: $5 / 5$
$137 / 137 \mathrm{SCl}$

114:23

Central neuropathic

pain

46/46 SCl

$42: 4$

Neuropathic

-

Tai et al. ${ }^{23}$

USA

Double-blind,

crossover RCT

Jadad: $5 / 5$

To et al.27

Australia

Retrospective

D\&B: $27 / 44$

\section{$14 / 14 \mathrm{SCl}$ \\ 6:7 \\ Neuropathic}

28:10

Neuropathic

\section{Carbamazepine \\ Treatment: up to $600 \mathrm{mg}$ per day for $1 \mathrm{mo}$} and increasing doses through to week 3 . Decrease and discontinuation in 4th week. Control: up to $600 \mathrm{mg}$ per day placebo for 1 mo and increasing doses through to week 3. Decrease and discontinuation in 4th week. VAS, SF-36, Zung self-rating depression scale

Pregabalin.

Either flexible dose pregabalin 150-600 mg per day or placebo b.i.d for 12 wks. Daily pain diary; NRS.

\section{Gabapentin}

Treatment: 4-week course $(3 \times$ daily; progressively increased from $300-600 \mathrm{mg}$ per day) followed by 2 week washout period, and crossover of 4 wks medication/placebo. Control: 4 week course of placebo $(3 \times$ daily $)$ followed by 2-week washout period and crossover of 4 wks medication/placebo. NPS

Gabapentin

Retrospective chart review of patient's prescribed gabapentin for neuropathic pain. VAS or verbal descriptions.

Could not confirm utility of carbamazepine in preventing occurrence of neuropathic pain.

At end of mo 1 , there was a statistically significant difference $(P=0.024)$ for moderate/intense pain between 2 groups. There was no significant difference at mo 3 and 6.

No difference in occurrence of at or below level NP between the 2 groups at mo 3 and 6 .

Treatment superior to placebo on pain intensity $(P=0.001)$. Week 1 treatment group scores significantly lower and remained low.

Mean treatment difference between incomplete injury groups was $1.79(0.9,2.7 ; P<0.001)$ and $1.25(0.1$ 2.2; $P<0.05)$ for complete.

The proportions of patient with a $>-30 \%$ reduction and a $>-50 \%$ reduction in pain score from baseline at endpoint were significantly higher in treatment group than control group.

At endpoint, $15.9 \%$ patients in treatment group had severe pain compared with $43.3 \%$ in control. Over $1 / 3$ patients in treatment group had no/mild pain at endpoint compared with $10.5 \%$ in control group.

Significant reduction in 'unpleasant feeling' with gabapentin compared to placebo $(P=0.028)$.

Trends of reductions with gabapentin vs placebo for 'pain intensity' $(P=0.094)$ and 'burning feeling' $(P=0.065)$. No other differences for any other pain descriptors.

$76 \%$ had some improvement from gabapentin therapy. 9 patients ceased therapy within first month due to dizziness, somnolence, ineffectiveness.

Statistically significant difference found between times $(P=0.001)$ with a significant curvilinear trend $(P=0.001)$. Large difference pre and 1 mo post with no significant difference between post periods (1, 3, $6 \mathrm{mo})$. Most improvement occurred within first month.

Mean pain intensity scores before and after 4 wks of treatment changed from 7.4 (SD 1) to 7.3 (SD 2) in the placebo group; and from 7.6 (SD 0.8) to 5.1 (SD 2.9) in the treatment group.

Treatment: Started with 1 capsule per day. Escalating doses if relief insufficient (150, 300 or $600 \mathrm{mg}$ per day).

Control: Started with 1 capsule per day placebo.

If relief insufficient, titrated to higher dose (150, 300 or 600 mg per day).

VAS; PDI
Statistically significant mean pain endpoint for treatment compared with placebo: 2.18 with $95 \% \mathrm{Cl}$ $0.57-3.80 P=0.01$. No difference in pain relief following treatment between group with NP as a result of brain injury and NP as a result of $\mathrm{SCl}$.

Abbreviations: B.I.D, twice daily; CESD-SF, Centre for Epidemiologic Studies Depression Scale — Short Form; DAAC, duration adjusted average change; D\&B, Downs \& Black; M:F, ratio of males to females; Mo, months; MPQ, McGill Pain Questionnaire; NPS, numeric pain scale; NRS, numeric rating scale; PDI, pain disability index; SCI, spinal cord injury; VAS, visual analog scale; Wks, weeks.

As a result, an effectiveness analysis was run. The criteria used to interpret the resulting effect sizes were those outlined in Cohen (1988). ${ }^{14}$

\section{RESULTS}

\section{Study design and quality}

A comparison of a treatment and control group was undertaken in 10 studies. ${ }^{15-24}$ The most popular design for intervention studies which met inclusion criteria was the double-blind crossover trial $(n=4),{ }^{16,18,19,23}$ followed by the double-blind parallel-group trial $(n=3) .^{21,22,24}$ One study had a double-blind, triple crossover RCT design. ${ }^{20}$ Other designs included: 1 pre-post study, ${ }^{25} 1$ longitudinal observational study ${ }^{26}$ and 1 retrospective chart review. ${ }^{27}$ See Table 1 for a summary of included studies and methodological quality of each article. Power calculations were provided in 7 studies. ${ }^{15,18-22,24}$ Sample size ranged from $14^{23}$ to $219^{15}$ participants.

Gabapentinoids were examined in $9^{15,19,20,22-27}$ of the 13 studies. The following drugs were examined in the remaining studies: valproate, ${ }^{16}$ lamotrigine, ${ }^{17}$ levetiracetam, ${ }^{18}$ and carbamazepine. ${ }^{21}$ All studies used an oral route of administration.

\section{Participant characteristics}

Baseline characteristics were generally well described in all included studies. For instance, age was provided in all but one study, ${ }^{26}$ age ranged from a mean of 32.5 years ${ }^{16}-54.7$ years. ${ }^{24}$ Sex categorization of the sample was reported in all 13 studies-ratio of male to female participants can be seen in Table 1 with a few studies including almost double the number of males compared to females. Information on 
completeness of injury was provided in 7 studies. ${ }^{15,17,18,21,24-26}$ Number of participants with complete injury ranged from $5^{26}-106,{ }^{15}$ while number of participants with incomplete injury ranged from $10^{17,24}-105 .^{15}$ Level of injury (tetraplegia or paraplegia) was reported in 7 of the 13 studies; ${ }^{16,20,22,23,25-27}$ with a range of $4^{16}-58^{22}$ participants with tetraplegia and $6^{23}-79^{22}$ for participants with paraplegia. Time since injury was recorded in 5 of the 13 studies, ${ }^{17,19,20,22,23}$ with a duration range of 1 month-396 months post injury. The cause of spinal cord injury was reported in 4 of the 13 studies; ${ }^{15,18,21,23,25}$ these included: hemorrhage, surgery, infection, tumor, firearm and other weapons, falls, prolapsed disk, sports, diving, transverse myelitis, ischemia and motor vehicle accidents. When reporting the type of pain being examined, Cardenas et al. ${ }^{15}$ was the only article to mention taxonomy, that is, the Bryce-Ragnarsson taxonomy. Onset of pain post injury was mentioned in 10 of the 13 studies ${ }^{15-18,20,22,23,25,27}$ with a duration range (when months/years are attributed to pain description) of $<6$ months-45 months.

\section{Effectiveness on primary outcome}

Effect size calculations were possible for 10 studies (Table 2), while the results of the remaining studies were descriptively provided in Table 1. Overall, 9 studies evaluated the effects of gabapentin and pregabalin on improving pain outcomes after SCI. Of these, 6 studies examined the effectiveness of gabapentin for pain after SCI. ${ }^{19,20,23,25-27}$ Four of these studies found large effect size (0.873-3.362) for improvement in pain relief ${ }^{19,23,25,27}$ with effects maintained for up to 24 weeks. Data to calculate an effect size was not available for Putzke et al. ${ }^{26}$; however, the study reported improvement in pain by 2 points or more after gabapentin treatment in 14 of 21 participants. Ahn et al. ${ }^{25}$ found gabapentin was effective in improving pain among those individuals with chronic pain ( $>6$ months) and those with more subacute pain ( $<6$ months) for up to 8 weeks. The only study using an active placebo (Benadryl) as the control intervention found no significant improvement in pain post gabapentin treatment. ${ }^{20}$ Pregabalin was shown to have moderate to large effect (0.695-3.805) on improving neuropathic pain after SCI in 3 studies. ${ }^{15,22,24}$

The remaining 4 studies examined less commonly utilized anticonvulsants in pain management after SCI. ${ }^{16-18,21}$ Drewes et al. ${ }^{16}$ found valproate was not effective in improving neuropathic pain after SCI when compared to control. Finnerup et al. ${ }^{17}$ reported lamotrigine was only effective in reducing neuropathic pain among individuals with incomplete lesions when compared to those with complete lesions $(d=1.374 \pm 0.258-2.489, \quad P<0.016)$ at 20 weeks post treatment. No significant improvement in pain was seen in participants with at and below level neuropathic pain with the administration of levetiracetam. ${ }^{18}$ Carbamazepine significantly improved pain among individuals with moderate to intense neuropathic pain at one month post treatment compared to the control group. ${ }^{21}$ However, these improvements were not sustained over a 3 and 6 month period.

\section{Secondary outcomes}

Sleep interference was shown to improve with the use of both gabapentin $^{25}$ and pregabalin; ${ }^{22}$ the use of lamotrigine showed no significant improvement in sleep interference. ${ }^{17}$ Two studies examined the effect of pregabalin on the Hospital Anxiety and Depression Scale (HADS) anxiety subscale and depression subscale. ${ }^{15,22}$ Cardenas et al. ${ }^{15}$ found treatment with pregabalin had a large effect size in improving both anxiety and depression at week 16; however, Siddall et al. ${ }^{22}$ found no effect at 12 weeks. Vranken et al. ${ }^{24}$ found significant improvement in EuroQOL (EQ-5D utility) post pregabalin treatment at week 4 but no significant improvement in the Pain Disability Index. For more details on secondary outcomes see Table 3.

\section{Adverse events}

Effect size data was available in 4 studies examining the adverse events of anticonvulsants. ${ }^{15,17,19,24}$ No significant difference in adverse event occurrence was noted between the control group and lamotrigine ${ }^{17}$ or gabapentin. ${ }^{19}$ However, Cardenas et al. ${ }^{15}$ found individuals

Table 2 Effect size data for primary outcome (pain intensity)

\begin{tabular}{|c|c|c|c|c|c|c|}
\hline Study & Treatment & Subgroup & Time point & Effect size & $95 \% \mathrm{Cl}$ & $\mathrm{P}$-value \\
\hline \multirow[t]{8}{*}{ Ahn et al. ${ }^{25}$} & \multirow[t]{8}{*}{ Gabapentin } & \multirow[t]{4}{*}{ Less than 6 months chronic pain } & Week 2 & 1.315 & $0.508-2.122$ & 0.001 \\
\hline & & & Week 4 & 2.335 & $1.194-3.476$ & 0.001 \\
\hline & & & Week 6 & 2.664 & $1.404-3.924$ & 0.0001 \\
\hline & & & Week 8 & 2.508 & $1.305-3.711$ & 0.0001 \\
\hline & & \multirow[t]{4}{*}{ More than 6 months chronic pain } & Week 2 & 0.873 & $0.258-1.489$ & 0.005 \\
\hline & & & Week 4 & 1.177 & $0.495-1.858$ & 0.001 \\
\hline & & & Week 6 & 1.153 & $0.477-1.829$ & 0.001 \\
\hline & & & Week 8 & 1.192 & $0.507-1.877$ & 0.001 \\
\hline Cardenas et al. ${ }^{15}$ & Pregabalin & N/A & Week 16 & 3.805 & $3.53-4.257$ & 0.0001 \\
\hline \multirow[t]{2}{*}{ Finnerup et al. ${ }^{17}$} & \multirow[t]{2}{*}{ Lamotrigine } & All participants & Week 20 & 0.529 & $-0.102-1.160$ & 0.100 \\
\hline & & Participants with incomplete injury & Week 20 & 1.374 & $0.258-2.489$ & 0.016 \\
\hline \multirow[t]{2}{*}{ Levendoglu et al. ${ }^{19}$} & \multirow[t]{2}{*}{ Gabapentin } & \multirow[t]{2}{*}{$\mathrm{N} / \mathrm{A}$} & Week 4 & 3.362 & $2.400-4.325$ & 0.0001 \\
\hline & & & Week 8 & 4.377 & $3.235-5.19$ & 0.0001 \\
\hline Rintala et al. ${ }^{20}$ & Gabapentin & $\mathrm{N} / \mathrm{A}$ & Week 8 & 0.096 & $-0.495-0.687$ & 0.750 \\
\hline Siddall et al. ${ }^{22}$ & Pregabalin & N/A & Week 12 & 0.695 & $0.349-1.041$ & 0.0001 \\
\hline Tai et al. ${ }^{23}$ & Gabapentin & N/A & Week 4 & 1.268 & $0.120-2.416$ & 0.030 \\
\hline \multirow[t]{3}{*}{ To et al. ${ }^{27}$} & \multirow[t]{3}{*}{ Gabapentin } & \multirow[t]{3}{*}{$\mathrm{N} / \mathrm{A}$} & Week 4 & 0.900 & $0.367-1.433$ & 0.001 \\
\hline & & & Week 12 & 1.128 & $0.458-1.798$ & 0.001 \\
\hline & & & Week 24 & 1.018 & $0.414-1.622$ & 0.001 \\
\hline Vranken et al..24 & Pregabalin & $\mathrm{N} / \mathrm{A}$ & Week 4 & 0.963 & $0.309-1.618$ & 0.004 \\
\hline
\end{tabular}


Table 3 Effect size data for secondary outcomes

\begin{tabular}{|c|c|c|c|c|c|c|c|}
\hline Study & Treatment & Outcome & Subgroup & Time point & Effect size & $95 \% \mathrm{Cl}$ & P-value \\
\hline \multirow[t]{8}{*}{ Ahn et al. ${ }^{25}$} & Gabapentin & Sleep interference & $\begin{array}{l}\text { Less than } 6 \text { months } \\
\text { chronic pain }\end{array}$ & Week 2 & 0.810 & $0.129-1.490$ & 0.02 \\
\hline & & & & Week 4 & 1.159 & $0.395-1.923$ & 0.003 \\
\hline & & & & Week 6 & 1.172 & $0.405-1.940$ & 0.003 \\
\hline & & & & Week 8 & 1.201 & $0.426-1.976$ & 0.002 \\
\hline & & & $\begin{array}{l}\text { More than } 6 \text { months } \\
\text { chronic pain }\end{array}$ & Week 2 & 0.503 & $-0.053-1.059$ & 0.076 \\
\hline & & & & Week 4 & 0.662 & $0.084-1.241$ & 0.025 \\
\hline & & & & Week 6 & 0.634 & $0.06-1.208$ & 0.030 \\
\hline & & & & Week 8 & 0.634 & $0.06-1.208$ & 0.030 \\
\hline \multirow[t]{2}{*}{ Cardenas et al. ${ }^{15}$} & Pregabalin & HADS anxiety & $\mathrm{N} / \mathrm{A}$ & Week 16 & 2.029 & $1.687-2.371$ & 0.0001 \\
\hline & & HADS depression & & Week 16 & 2.912 & $2.513-3.311$ & 0.0001 \\
\hline Finnerup et al. ${ }^{17}$ & Lamotrigine & Sleep interference & N/A & Week 20 & 0.141 & $-0.453-0.735$ & 0.642 \\
\hline \multirow[t]{3}{*}{ Siddall et al..22 } & Pregabalin & HADS anxiety & $N / A$ & Week 12 & 0.103 & $-0.233-0.440$ & 0.547 \\
\hline & & HADS depression & & Week 12 & 0.024 & $-0.312-0.360$ & 0.888 \\
\hline & & Sleep interference & & Week 12 & 0.446 & $0.106-0.786$ & 0.010 \\
\hline \multirow[t]{2}{*}{ Vranken et al. ${ }^{24}$} & Pregabalin & EQ-5D utility & $\mathrm{N} / \mathrm{A}$ & Week 4 & 1.060 & $0.398-1.722$ & 0.002 \\
\hline & & Pain disability Index & & Week 4 & 0.415 & $-0.211-1.042$ & 0.194 \\
\hline
\end{tabular}

Table 4 Odds ratios for Adverse Events

\begin{tabular}{|c|c|c|c|c|c|}
\hline Study & Treatment & Adverse Event & Odds Ratio & $95 \% \mathrm{Cl}$ & P-value \\
\hline \multirow[t]{7}{*}{ Cardenas et al. ${ }^{15}$} & \multirow[t]{7}{*}{ Pregabalin } & Blurred vision & 17.488 & $0.997-306.852$ & 0.050 \\
\hline & & Dizziness & 3.659 & $1.408-9.510$ & 0.008 \\
\hline & & Dry Mouth & 3.029 & $0.797-11.508$ & 0.104 \\
\hline & & Edema & 6.000 & $0.710-50.694$ & 0.100 \\
\hline & & Fatigue & 8.154 & $1.002-66.345$ & 0.050 \\
\hline & & Peripheral Edema & 4.552 & $1.259-16.458$ & 0.021 \\
\hline & & Somnolence & 3.277 & $1.650-6.508$ & 0.001 \\
\hline Finnerup et al. ${ }^{17}$ & Lamotrigine & Sleep & 1.291 & $0.440-3.792$ & 0.642 \\
\hline \multirow[t]{5}{*}{ Levendoglu et al. ${ }^{19}$} & \multirow[t]{5}{*}{ Gabapentin } & Dizziness & 3.353 & $0.318-35.364$ & 0.314 \\
\hline & & Edema & 8.200 & $0.396-169.899$ & 0.174 \\
\hline & & Headache & 1.000 & $0.058-17.181$ & 1.000 \\
\hline & & Sedation & 8.200 & $0.396-169.899$ & 0.174 \\
\hline & & Weakness & 3.000 & $0.507-17.740$ & 0.226 \\
\hline \multirow[t]{6}{*}{ Vranken et al. ${ }^{24}$} & \multirow[t]{6}{*}{ Pregabalin } & Cognitive performance & 0.643 & $0.174-2.381$ & 0.508 \\
\hline & & Confusion & 2.154 & $0.515-9.000$ & 0.293 \\
\hline & & Dizziness & 1.256 & $0.334-4.733$ & 0.736 \\
\hline & & Nausea & 1.714 & $0.400-7.340$ & 0.468 \\
\hline & & Peripheral Edema & 0.211 & $0.021-2.079$ & 0.182 \\
\hline & & Somnolence & 1.000 & $0.288-3.476$ & 1.000 \\
\hline
\end{tabular}

receiving pregabalin were significantly more likely to experience dizziness (3.7 times), fatigue (8.2 times), peripheral edema (4.6 times) and somnolence (3.3 times) than those in the control group (see Table 4).

\section{DISCUSSION}

Thirteen studies examining the efficacy of anticonvulsants for pain relief post SCI were identified. The most commonly studied anticonvulsants are gabapentin and pregabalin which are recommended in clinical practice guidelines as first-line treatments. Research evidence is more limited on the remaining anticonvulsants: valproate, lamotrigine, levetiracetam and carbamazepine. Varying levels of effectiveness for gabapentinoids can be seen; 4 studies show large effect sizes (0.873-3.362) for pain improvement with gabapentin treatment alone and moderate to large effect sizes for pregabalin (0.695-3.805). The effectiveness of valproate, lamotrigine, levetiracetam and carbamazepine is less certain, given we had to rely on only one trial that met inclusion criteria for each. That being said, based on single studies, valproate and levetiracetam were not successful in improving pain, ${ }^{16,18}$ lamotrigine was effective at reducing neuropathic pain among individuals with incomplete lesions, ${ }^{17}$ and carbamazepine was found to be effective in individuals with moderate to intense neuropathic pain. $^{21}$ In studies which examined subgroups, lamotrigine and pregabalin were more effective in persons with incomplete injury. ${ }^{17,22}$ For those authors reporting adverse events, no significant difference was found in lamotrigine or gabapentin when compared to controls. In one study, pregabalin was associated with blurred vision, drowsiness, fatigue, peripheral edema and dizziness. ${ }^{15}$ 
Rintala et al. ${ }^{20}$ was the only study to find gabapentin was no more effective in relieving pain intensity than the placebo diphenhydramine at week 8 of the trial, and to use an active control. The authors state diphenhydramine was used as it has similar side effects to that of amitryptiline and gabapentin; however, it is not a pain reliever. This active placebo has a half-life of $3-12 \mathrm{~h}$ in comparison to gabapentin's $6-8 \mathrm{~h}$ and amitryptiline's $24-70 \mathrm{~h}$. This placebo was placed in capsules identical to the other 2 study medications. Two early crossovers were thought to be the result of possible side effects (palpitations, fatigue, dizziness and drowsiness) caused by the placebo. Bjorkedal and Flaten ${ }^{28}$ explored the concept of an active placebo (i.e., mimicking side effects of an active treatment) in reducing pain and found an increase in reduction in pain intensity. This within-subjects balanced placebo design tested the hypothesis that expectations and responses to a placebo (in this case, caffeine) can be enhanced by drug side effects. Results showed using an active placebo versus an inert placebo can produce differing treatment effects. Future studies are best conducted using an active placebo to establish effectiveness of these medications.

Gabapentinoids were found to have a positive effect on sleep interference. 22,25 Two factors accounting for this appear to be a reduction of pain levels and drug side effects. Both pregabalin and gabapentin can lead to fatigue, sleepiness, sedation and somnolence. ${ }^{15,18,19,24}$ Cardenas et al. ${ }^{15}$ and Levendoglu et al. ${ }^{19}$ found the treatment group was 3.3 and 8 times, respectively, more likely to experience sedation than the placebo control group.

\section{Current trials}

Three relevant trials were retrieved through our grey literature database search. Green ${ }^{29}$ is conducting a clinical trial in Spain examining the efficacy of pregabalin for the treatment and prevention of neuropathic pain as a result of SCI. This proposed study is a randomized, double-blind, crossover trial comparing pregabalin treatment with a placebo. The primary outcome measure is daily at-level pain intensity as measured by a numerical rating scale (0-10). A Canadian double-blind, randomized, placebo-controlled trial is testing the efficacy of pregabablin in the prevention of neuropathic pain post SCI across a rehabilitation center setting. ${ }^{30} \mathrm{~A}$ study headed by Pfizer Japan is assessing pregabalin for the treatment of central neuropathic pain in a non-randomized, single group, openlabel trial. $^{31}$

\section{Strengths and limitations}

This systematic review attempts to collate all available data regarding the effectiveness of anticonvulsant medications for the relief of pain post spinal cord injury. Notably, we have not restricted interventional studies to RCTs but have also included other study designs. Quality of methodological design of interventional studies has been assessed using validated tools. Outcome measures proved to be too heterogeneous to be compared and as a result the data could not be pooled. However, effect sizes and odds ratio were calculated.

\section{Recommendations for future research}

It appears well established that gabapentin and pregabalin are the two anticonvulsants which have been shown to have some limited benefit. The role of combination treatment may improve the analgesic effect of these medications. Studies comparing different anticonvulsants, particularly for some subgroups, may be helpful. The utility of less commonly prescribed anticonvulsants has not yet adequately been established.
Given evidence showing a difference in treatment effect may exist when using an active placebo design versus an inert placebo design, it would be beneficial to incorporate an active placebo when testing the effect of anticonvulsants in relieving pain post spinal cord injury. Amongst the 10 RCTs reviewed, only one study utilized an active placebo design.

Amongst all the anticonvulsants included in this review, the strongest evidence for the management of pain post SCI points to gabapentioids. Varying levels of the effectiveness of gabapentinoids can be seen, with 4 trials showing large effect sizes. Being cognizant of the very limited work done with other anticonvulsants, carbamazepine and lamotrigine were found to be effective in relieving pain post SCI. The lesser-studied anticonvulsants merit further investigation.

\section{DATA ARCHIVING}

There were no data to deposit.

\section{CONFLICT OF INTEREST}

The authors declare no conflict of interest.

\section{ACKNOWLEDGEMENTS}

This work was supported by Rick Hansen Institute and Ontario Neurotrauma Foundation.

1 Donnelly C, Eng JJ. Pain following spinal cord injury: the impact on community reintegration. Spinal Cord 2005; 43: 278-282.

2 Widerström-Noga EG, Felipe-Cuervo E, Yezierski RP. Chronic pain after spinal injury: interference with sleep and daily activities. Arch Phys Med Rehabil 2001; 82: 1571-1577.

3 Barrett H, McClelland JM, Rutkowski SB, Siddall PJ. Pain characteristics in patients admitted to hospital with complications after spinal cord injury. Arch Phys Med Rehabil 2003; 84: 789-795.

4 Jensen MP, Hoffman AJ, Cardenas DD. Chronic pain in individuals with spinal cord injury: a survey and longitudinal study. Spinal Cord 2005; 43: 704-712.

5 Heutink M, Post MW, Wollaars MM, van Asbeck FW. Chronic spinal cord injury pain: pharmacological and non-pharmacological treatments and treatment effectiveness. Disabil Rehabil 2011; 33: 433-440.

6 Attal N, Mazaltarine G, Perrouin-Verbe B, Albert TSOFMER. Chronic neuropathic pain management in spinal cord injury patients. What is the efficacy of pharmacological treatments with a general mode of administration? (oral, transdermal, intravenous). Ann Phys Rehabil Med 2009; 52: 124-141.

7 Siddall PJ, Middleton JW. A proposed algorithm for the management of pain following spinal cord injury. Spinal Cord 2006; 44: 67-77.

8 Baron R. Neuropathic pain: a clinical perspective. Handb Exp Pharmacol 2009; 194: 3-30.

9 Finnerup NB, Jensen TS. Spinal cord injury pain - mechanisms and treatment. Eur J Neurol 2004; 11: 73-82.

10 Siddall PJ. Management of neuropathic pain following spinal cord injury: now and in the future. Spinal Cord 2009; 47: 352-359.

11 Moulin DE, Clark AJ, Gilron I, Ware MA, Watson CPN, Sessle BJ et al. Pharmacological management of chronic neuropathic pain - consensus statement and guidelines from the Canadian Pain Society. Pain Res Manag 2007; 12: 13-21.

12 Jadad AR, Moore RA, Carroll D, Jenkinson C, Reynolds DJ, Gavaghan DJ et al. Assessing the quality of reports of randomized clinical trials: is blinding necessary? Control Clin Trials 1996; 17: 1-12.

13 Downs SH, Black N. The feasibility of creating a checklist for the assessment of the methodological quality both of randomised and non-randomised studies of health care interventions. J Epidemiol Community Health 1998; 52: 377-384.

14 Cohen J. Statistical power and analysis for behavioral sciences, 2nd ed. New York, 1988.

15 Cardenas DD, Nieshoff EC, Suda K, Goto SI, Sanin L, Kaneko T et al. A randomized trial of pregabalin in patients with neuropathic pain due to spinal cord injury. Neurology 2013; 80: 533-539.

16 Drewes AM, Andreasen A, Poulsen LH. Valproate for treatment of chronic central pain after spinal cord injury: a double-blind cross-over study. Paraplegia 1994; 32: 565-569.

17 Finnerup NB, Sindrup SH, Bach FW, Johannesen IL, Jensen TS. Lamotrigine in spinal cord injury pain: a randomized controlled trial. Pain 2002; 96: 375-383.

18 Finnerup NB, Grydehøj J, Bing J, Johannesen IL, Biering-Sørensen F, Sindrup SH et al. Levetiracetam in spinal cord injury pain: a randomized controlled trial. Spinal Cord 2009; 47: 861-867. 
19 Levendoglu F, Ögün C, Özerbil O, Ögün TC, Ugurlu H. Gabapentin is a first line drug for the treatment of neuropathic pain in spinal cord injury. Spine 2004; 29 $743-751$.

20 Rintala DH, Holmes SA, Courtade D, Fiess Tastard LV, Loubser PG. Comparison of the effectiveness of amitriptyline and gabapentin on chronic neuropathic pain in persons with spinal cord injury. Arch Phys Med Rehabil 2007; 88: 1547-1560.

21 Salinas FA, Lugo LH, García HI. Efficacy of early treatment with carbamazepine in prevention of neuropathic pain in patients with spinal cord injury. Am J Phys Med Rehabil 2012; 91: 1020-1027.

22 Siddall PJ, Cousins MJ, Otte A, Griesing T, Chambers R, Murphy TK. Pregabalin in central neuropathic pain associated with spinal cord injury: a placebo-controlled trial. Neurology 2006; 67: 1792-1800.

23 Tai Q, Kirshblum S, Chen B, Millis S, Johnston M, DeLisa JA. Gabapentin in the treatment of neuropathic pain after spinal cord injury: a prospective, randomized, double-blind, crossover trial. J Spinal Cord Med 2002; 25: 100-105.

24 Vranken JH, Dijkgraaf MGW, Kruis MR, van der Vegt MH, Hollman MW, Heesen M. Pregabalin in patients with central neuropathic pain: a randomized, double-blind, placebo-controlled trial of a flexible-dose regimen. Pain 2008; 136: 150-157.
25 Ahn S, Park H, Lee B, Moon HW, Jang SH, Sakong J et al. Gabapentin effect on neuropathic pain compared among patients with spinal cord injury and different durations of symptoms. Spine 2003; 28: 341-347.

26 Putzke JD, Richards JS, Kezar L, Hicken BL, Ness TJ. Long-term use of gabapentin for treatment of pain after traumatic spinal cord injury. Clin J Pain 2002; 18: 116-121.

27 To T, Lim TC, Hill ST, Moon HW, Jang SH, Sakong J et al. Gabapentin for neuropathic pain following spinal cord injury. Spinal Cord 2002; 40: 282-285.

28 Bjørkedal E, Flaten MA. Interaction between expectancies and drug effects: an experimental investigation of placebo analgesia with caffeine as an active placebo. Psychopharmacology (Berl) 2011; 215: 537-548.

29 Green JT. Pregabalin for the Treatment and Prevention of Spinal Cord Injury Neuropathic Pain. URL: http://ClinicalTrials.gov/show/NCTO1479556 (accessed 04/22 2013).

30 Short CA. Can Pregabalin Prevent the Development of Neuropathic Pain Following Spinal Cord Injury? URL: http://clinicaltrials.gov/show/NCT00879021 (accessed 04/ 22 2013).

31 Pfizer Inc. An Open-Label Long-Term Study Of Pregabalin For The Treatment Of Central Neuropathic Pain (Post Spinal Cord Injury Pain, Post Stroke Pain, And Multiple Sclerosis Pain). URL: http://clinicaltrials.gov/show/NCT01202227 (accessed 04/22 2013).

Supplementary Information accompanies this paper on the Spinal Cord website (http://www.nature.com/sc) 